\title{
Effective Evaluation of XML Queries in a Visual Environment
}

\author{
Weimin $\mathrm{He}$ \\ Department of Computing and New Media Technologies \\ University of Wisconsin-Stevens Point \\ Stevens Point, WI, USA 54481 \\ whe@uwsp.edu \\ Teng LV \\ School of Information Engineering \\ Anhui Xinhua University \\ Hefei, Anhui, China 230031 \\ It0410@163.com \\ Ping Yan \\ School of Science \\ Anhui Agricultural University \\ Hefei, Anhui, China 230031 \\ want2y2002@163.com
}

\begin{abstract}
Since its emergence in 1997, Extensible Markup Language (XML) has become a standard data format for data exchange among a variety of web applications. In this paper, we present a framework to effectively evaluate $X M L$ queries in a visual environment. We have developed a system termed VXPath, which is a visual $X P a t h$ query evaluator that allows the user to evaluate various XPath queries by clicking the nodes in an expanding tree instead of typing the whole XPath queries manually. Our system GUI is built on top of Microsoft .NET Windows Forms Applications. We have implemented most XPath axes in our system, including child, descendant, self, parent, ancestor, following-sibling, preceding-sibling, predicate and so on. In order to handle large size XML documents, instead of loading the whole $X M L$ document into memory, we developed an effective algorithm to extract a concise data synopsis called structural summary from the original XML document to avoid the loading overhead for large XML documents. We evaluated our system over the data from XMark and DBLP and our system can handle large $X M L$ documents up to gigabytes.
\end{abstract}

Subject Categories and Descriptors: [H.2.3 Languages]; Query languages: [H.2.4 Systems]; Query processing

General Terms: Query languages, XML, Databases

Keywords: Database, XML, XPath, Query Processing
Received: 17 October 2018, Revised 3 January 2019, Accepted 16 January 2019

Review Metrics: Review Scale: 0/6, Review Score: 4.8/6, Interreviewer consistency: $82.5 \%$

DOI: $10.6025 / j \operatorname{dim} / 2019 / 17 / 3 / 113-121$

\section{Introduction}

Since its emergence in 1997, Extensible Markup Language $(\mathrm{XML})$ has become a standard data format for data exchange among a variety of web applications. A volume number of web data sources are represented as XML documents or fragments on the Internet. A large number of web services are also encoded in XML format. XML has undoubtedly become the new standard for data representation and data exchange on the web. Therefore, querying XML data on the web has attracted much attention in the database literature.

Khalifa et al [1] proposes bulk algebra called TIX, which integrates simple IR scoring schemes into a traditional pipelined query evaluator for an XML database. More specifically, new operators and efficient access methods are proposed to generate and manipulate scores of XML fragments during query evaluation. TeXQuery [2] supports a powerful set of fully composable full-text search primi- 
tives, which can be seamlessly integrated into the XQuery language. It mostly focuses on the TeXQuery language design and the underlying formal model, but also provides a costing model [5]. In [3], the authors present a framework that relaxes a full-text XPath query by dropping some predicates from its closure and scoring the approximate answers using predicate penalties. They further propose novel scoring methods by extending $T F * I D F$ ranking to account for both structure and content while considering query relaxations [6]. Sigurbjornsson et al, [22] propose a framework that decomposes the query into several pathterm pairs, evaluates these pairs separately and produces a final ranking that takes into account the scores of different sources of evidence. XRank [15] extends Google-like keyword search to XML. The authors propose an algorithm for scoring XML elements that takes into account both hyperlink and containment edges. New inverted list index structures based on Dewey IDs and associated query processing algorithms are also presented. XSEarch [12] is a semantic search engine that extends simple keyword search by incorporating keyword context information into the query, i.e., each query term is a keywordlabel pair instead of a single keyword. XSEarch extends the $T F^{*} I D F$ ranking scheme to rank the results. XKSearch [23] introduces the concept of smallest lowest common ancestors (SLCAs) and proposes two efficient algorithms, Indexed Lookup Eager and Scan Eager, for keyword search in XML documents according to the SLCA semantics.

In this paper, we propose a framework to evaluate XPath queries over XML documents in a user-friendly visual manner. we developed a prototype software system named VXPath to facilitate the efficient evaluation of XPath queries over XML data. VXPath is a visual XPath query evaluator that allows the user to evaluate an XPath query by clicking the nodes in an expanding tree instead of typing the whole XPath query by hand. Our system supports a variety of XPath axes, including child, descendant, self, parent, ancestor, following sibling, preceding-sibling, predicate and so on. In order to handle XML documents in very large size, instead of loading the whole XML document into memory, we extracted a concise data synopsis termed structural summary from the original XML document to avoid the loading overhead for large XML document. By looking at the structural summary in the graphical user interface, the user can obtain essential schema knowledge and issue an effective XPath query. This can greatly facilitate the learning and evaluation of XPath queries.

The rest of the paper is organized as follows. In section II, the algorithms for tree view binding is presented. Then we present the graphical user interface of VXPath and XPath evaluation algorithm in section III. In section IV, we illustrate an example XML document, the structural summary extracted from the document in order to handle XML documents in very large size, and the structural summary extraction algorithm. We report experimental results in section V. Finally, we conclude the paper and present the future work in section VI.

\section{Binding DOM Tree to Windows Treeview}

In order to display the content of an XML document in a visual manner, we leverage the DOM parser to create a DOM tree in memory and develop an algorithm to bind the DOM tree to a TreeView control in Windows Forms application. The key method for tree view binding written in $\mathrm{C \#}$ is shown in Figure 1. The first parameter of the method represents a node in the DOM tree. The second parameter represents a node in the TreeView. If the current XML node has children nodes, the program loops through the XML nodes until the leaf is reached and add the nodes to the TreeView during the looping process. If the current node has no children nodes, it can be a text node or empty element. Either case is processed separately in the algorithm.

\section{VXPath Graphical User Interface and XPath Query Evaluation}

The graphical user interface (GUI) of VXPath is shown in Figure 2. The GUI of VXPath consists of four main components. On the left panel, the user can specify the type of XPath queries and choose whether to match attributes in the query. In the middle section of the left panel, the user can choose the XPath axis he/she wants to evaluate when the user clicks a node in the tree view located in the central panel. The variety of types of axis supported in the our system are listed in Table I. When the user chooses an axis and clicks a node in the tree view in the central panel, the visual query result will be displayed as a tree view located at the bottom section of the left panel. For example, if the user chooses the descendant axis and clicks the node book in the tree view of the central panel, the descendant list of node book is listed as a tree view on the left. The user can further expand a node in the result list. In the central panel, there are two tabs. One tab named XML shows the structural summary of an XML document is displayed as an expanded tree. The user can click the nodes in the tree to form an XPath query. Another tab displays the query result of an XPath query. On the top panel is the XPath query expression created from the user clicks. On the top right is a button to run the XPath query. By clicking the File menu on the top left, the user can choose the XML document he/she wants to process.

In order to avoid the memory overhead to evaluate XPath queries over large XML documents in gigabytes, we exploited the XML SAX parser named XPathReader [32] from Microsoft to read XML data in a streaming manner instead of loading the whole document as a DOM tree. The $X P a t h R e a d e r$ provides the ability to filter and process large XML documents in an efficient manner using an XPathaware XmIReader. With the XPathReader, one can sequentially process a large document and extract an identified sub-tree matched by an XPath expression [32]. Our algorithm to evaluate XPath queries is shown in Figure 3. 
private void AddNode(XmlNode inXmlNode, TreeNode inTreeNode)

\{

XmlNode xNode;

TreeNode tNode;

XmlNodeList nodeList;

int i;

//Loop through the XML nodes until the leaf is reached.

/ / Add the nodes to the TreeView during the looping process.

if(inXmlNode.HaschildNodes)

\{

nodeList $=$ inXmlNode. ChildNodes;

for $(\mathrm{i}=0 ; \mathrm{i}<=$ nodeList. Count $-1 ; \mathrm{i}++)$

\{

xNode $=$ inXmlNode. childNodes;

String atts ="';

if(xNode.Attributes!=null)

\{

// how to get attributes as elements!

for $($ int $j=0 ; j<x N o d e$. Attributes.Count; $j++$ )

\{
atts+=“@

$"=\backslash " ”+x$ xode.Attributes.[j].Value $+"=\backslash " ” ;$

\}

inTreeNode.Nodes.Add(newTreeNode(xNode.Name + atts)); tNode $=$ inTreeNode.Nodes $[\mathrm{i}]$;

AddNode(xNode, tNode);

\} \}

else// no children, usually a text node, but could be an empty element

\{

String atts $=$ "”;

if(inXmlNode.Attributes!= null)

\{

for (int $\mathrm{j}=0 ; \mathrm{j}<\mathrm{inXmlNode.Attributes.Count;} \mathrm{j}++$ )

\{

atts + = “@” + inXmlNode.Attributes.[j].Name+

"=`" + inXmlNode.Attributes.[j].Value + “\”";

\}

inTreeNode. Text $=($ inXmlNode.Name + atts $) \cdot$ Trim ();

if(inXmlNode.InnerText ==" ")

inTreeNode.Nodes.Add(“")

\}

else

\{

inTreeNode. Text $=($ inXmlNode. OuterXml).Trim();

if (inXmlNode.InnerText $=="$ ")

inTreeNode.Nodes.Add(“");

\}

\}

Figure 1. Algorithm for Tree View Binding 


\begin{tabular}{|c|c|}
\hline Types Of Xpath QueryAxis & Meaning of XPath Query Axis \\
\hline Child & Selects nodes that are immedite child nodes of the Context Node \\
\hline Descendant & Selects all the descendant nodes of the context node \\
\hline Self & Selects the context node \\
\hline parent & Select the parent Node of the context node \\
\hline Ancestor & Select all the ancestor nodes of the context node \\
\hline Following- Siblings & Selects the next immediate sibling node of thew the context node \\
\hline Preceding-Sibling & Selects the previous immediate sibling node of the context node \\
\hline
\end{tabular}

Table 1. Types of Supported Xpath Axis

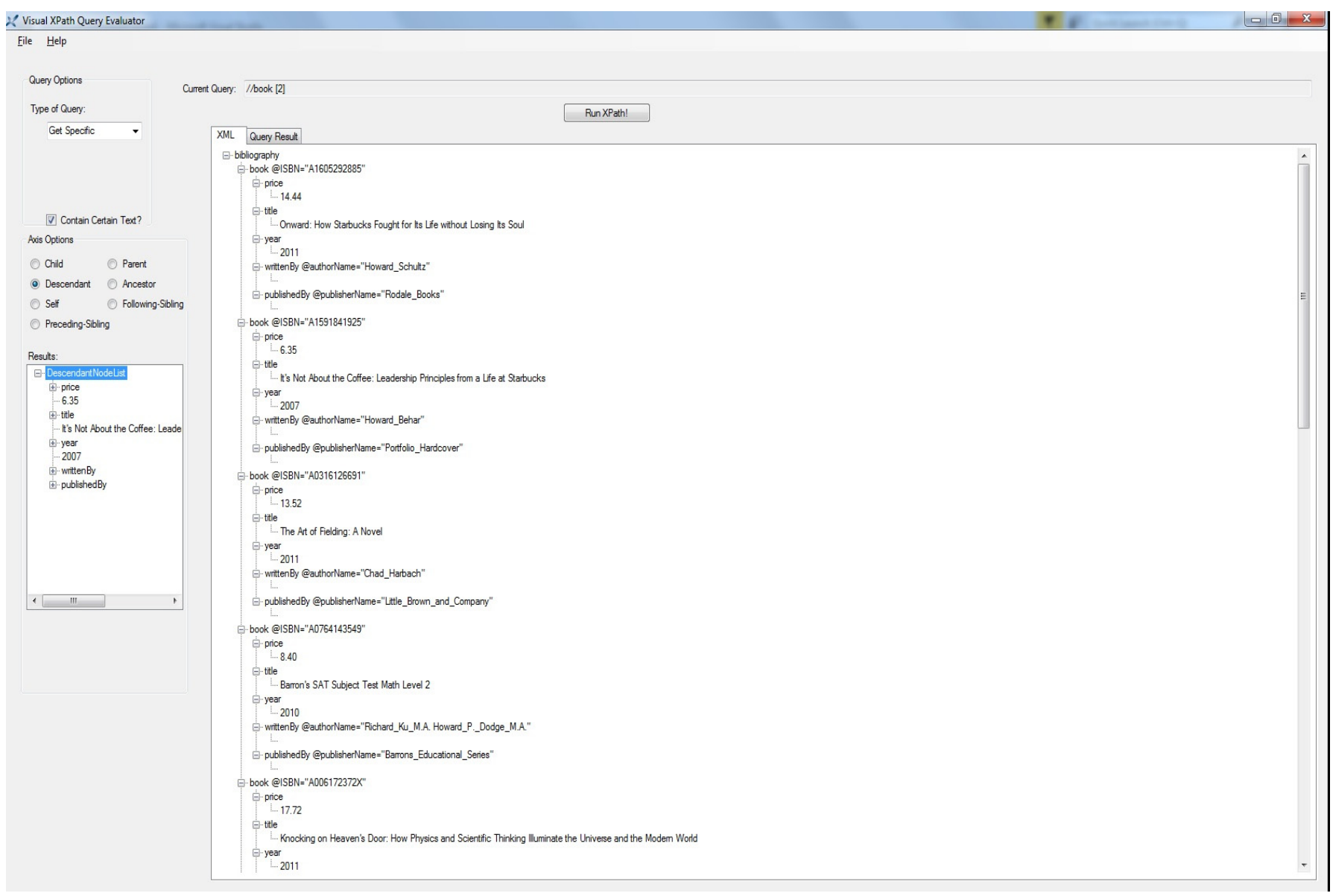

Figure 2. VXPath Graphical User Interface

\section{Handling of Large Size XML Documents}

To present an XML document to the user as a tree view, we exploit the DOM API in the .NET library to parse the document and construct a DOM tree in memory. However, this approach has a limitation because it can only handle smaller XML documents due to the fact that the size of a DOM tree grows very fast when the size of the $\mathrm{XML}$ document increases. In order to handle an XML document in large size, we extract a concise data synopsis called Structural Summary from an XML document, which is a structural markup that captures all the unique paths in the document. Instead of loading the original XML document into memory, we load and present the structural summary to the user. To achieve this goal, we leverage a third party SAX parser named SAX for .NET [31] to parse an $\mathrm{XML}$ document. Figure 4 gives an example XML document that represents the excerpted book information from Amazon. The structural summary of the example XML document is shown in Figure 5. Each node in an SS has a tagname and a unique Id. Note that one SS node may correspond to more than one elements in the original XML document. For example, the node book in Figure 5 corresponds to three book elements in Figure 4. We devel- 
private String evaluateXPath(String xpath, String path)

\{

if (xmlLarge) //Handling large $\mathrm{xml}$ documents

\{

FileStream strmTemp = new FileStream (path, FileMode.Open, FileAccess.Read);

XmlTextReader $x t r=$ new XmlTextReader(strmTemp);

xtr.DtdProcessing = Dtdprocessing. Ignore;

xtr.XmlResolver = null;

$\mathrm{XPathCollection} \mathrm{xc}=$ new XPathCollection();

xc.Add(xpath);

XpathReader $\mathrm{xpr}=$ new XPathReader $(\mathrm{xtr}, \mathrm{xc})$;

return EvaluateLarge(xpr);

\}

else

\{

XmlDocument doc = new XmlDocument();

doc.Load(path);

XPathNavigator navigator $=$ doc. CreateNavigator();

XPathExpression myXpath = XPathExpression.Compile(xpath);

return Evaluate(myXPath, navigator);

\}

\}

// Evaluate XPath Queries over Large XML Documents private static String EvaluateLarge(XPathReader xpr)

\{

StringBuilder results $=$ new StringBuilder();

\{

while(xpr.ReadUntilMatch())

results.Append(xpr.ReadOuterXm1());

\}

String fResults $=$ " $<$ results $>$ " results.ToString ()$+"</$ results $>$;

return fResults;

\}

Private static String Evaluate(XPathExpression expression, XPathNavigator navigator)

\{

String results $=$ "”;

XPathNodeIterator values $=($ XPath NodeIterator $)$ navigator . Evaluate (expression $)$; while(values.MoveNext())

\{

result $+=($ String)values.Current. OuterXml;

\}

result $=$ "<results $>$ " results $+"</$ results $>" ;$

return results;

\}

Figure 3. Algorithm for XPath Query Evaluation 


\begin{tabular}{|l|c|}
\hline XPath Query Expression & Query Evaluation Time (s) \\
\hline //site/regions & 16.66 \\
\hline //site/regions/samerica/item & 7.58 \\
\hline //site/regions/samerica/item/description/parlist & 3.78 \\
\hline //site/catgraph & 3.47 \\
\hline //site/regions/samerica/item/mailbox & 4.13 \\
\hline
\end{tabular}

Table 2. Experimental Evaluation Over Xmark Data

\begin{tabular}{|l|c|}
\hline XPath Query Expression & Query Evaluation Time (s) \\
\hline //dblp/article & 34.12 \\
\hline$/ / \mathrm{dblp} /$ book & 9.76 \\
\hline$/ / \mathrm{dblp} /$ article/editor & 8.15 \\
\hline$/ / \mathrm{dblp} /$ article/title & 13.78 \\
\hline$/ / \mathrm{dblp} /$ book/publisher & 5.32 \\
\hline
\end{tabular}

Table 3. Experimental Evaluation Over DBLP Data

oped a novel algorithm to extract the structural summary from the document. The excepted C\# code for structural summary extraction is shown in Figure 6.

\section{Experimental Result}

We conducted some experiments to evaluate our system over the XML data from XMark [26] and DBLP [30]. We first evaluated 5 XPath queries shown in Table 2 over the $\mathrm{XML}$ document from XMark. The file size is $113 \mathrm{MB}$. The file parsing time is 3.65 seconds. The XPath queries and the query evaluation time are shown in Table 2 . We then evaluated our system against the XML document from DBLP whose size is around 1GB. VXPath can handle this large XML document smoothly and the file parsing time is 58.42 seconds. The XPath queries and query evaluation time are shown in Table 3. As we can see, although the file size of DBLP is about 10 times of the file size of XMark, the query evaluation time does not grow linearly when the file size is larger.

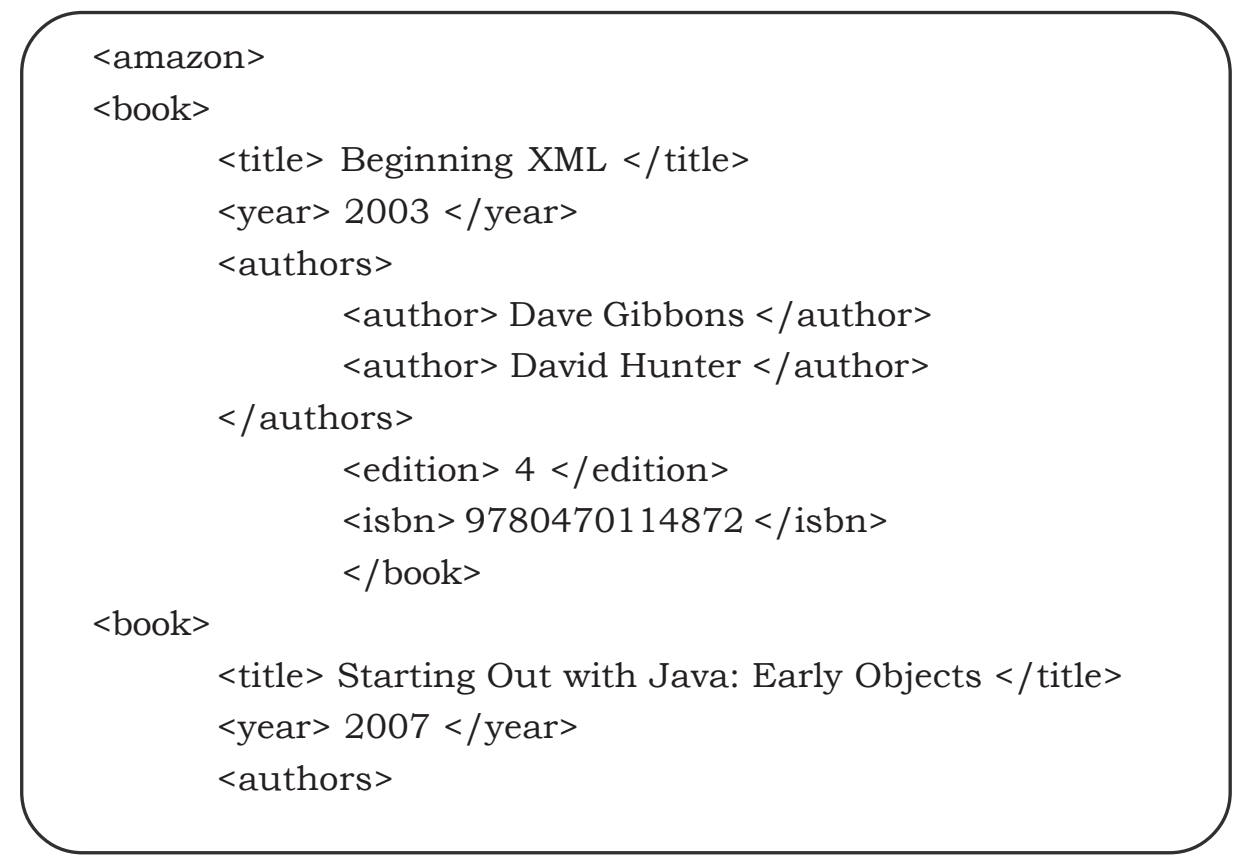




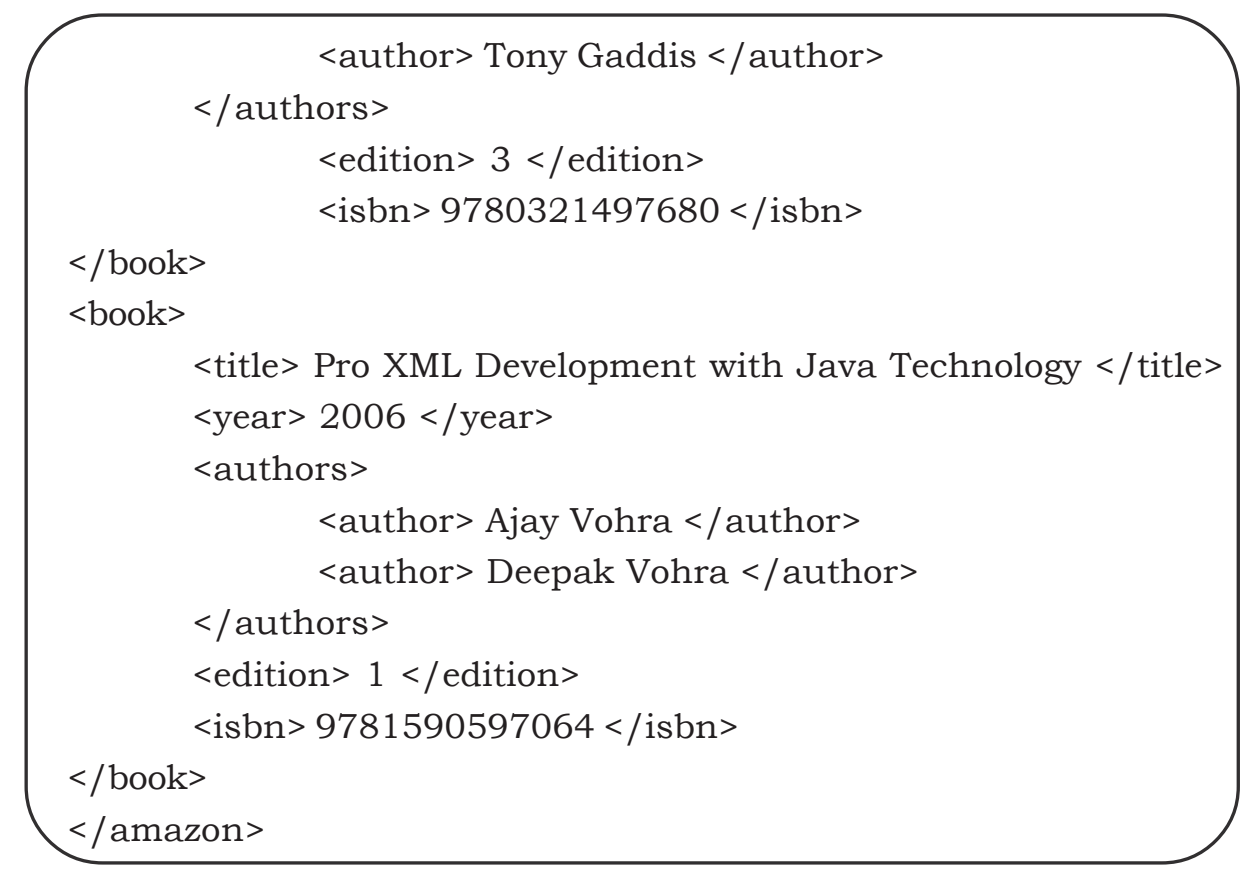

Figure 4. Example XML Document

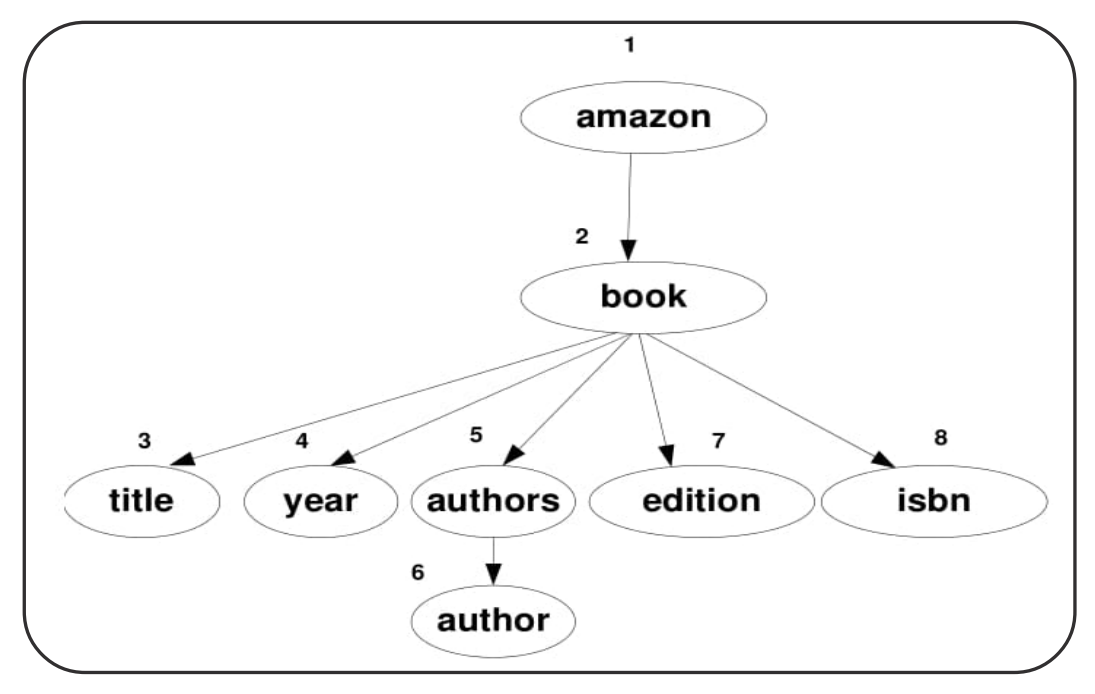

Figure 5. Structural Summary of Example XML Document

public class StructuralSummaryHandler : DefaultHandler \{

private int level;

public StructuralSummary[] summary;

int nodeId;

public StructuralSummaryHandler();

\{

level $=0$;

summary = new StructuralSummary[100];

nodeId $=0$;

summary $[0]=$ new StructuralSummary $(0, “ ")$;

\}

public override void StartElement(String Uri, StringLocalName, String qName, IAttributes atts)\{ Boolean found = false; 


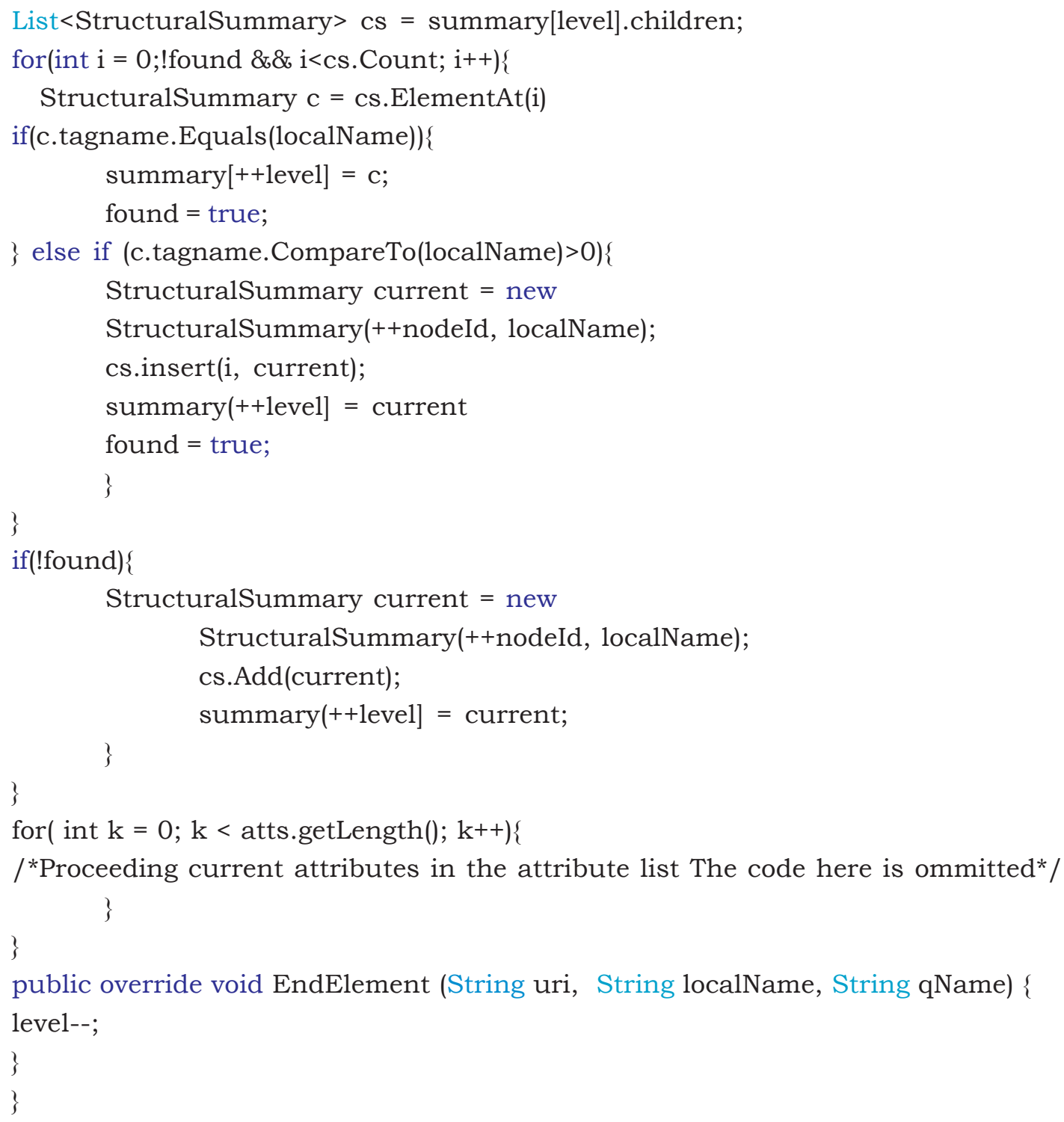

Figure 6. Extraction of Structural Summary

\section{Conclusion and Future Work}

In this paper, we proposed a new framework that can both evaluate XPath queries over XML data in a user-friendly visual manner and process XML documents in large size. Our system can support the visual evaluation of most XPath queries including forward and backward axes. This research work serves several purposes. First, The prototype system can serve as the foundation for the construction of the query engine in native XML databases because it can handle XML documents in any size. Secondly, the final completed software system can be used as a teaching/learning tool for the instructors/students in XML-related courses. The students can click around an XML file and learn how to construct an XPath expression in real time. This is much easier for the students beginning XML. As our future work, we plan to evaluate our system over some XML data sets from real world. We also plan to improve our software system to make it be able to handle XML document in larger size.

\section{References}

[1] Al-Khalifa, S., Yu, C., Jagadish, H. V. (2003). Querying Structured Text in an XML Database, In: Proceedings of SIGMOD'03, 2003.

[2] Amer-Yahia, S., Botev, C., Shanmugasundaram, S. (2004). TeXQuery: A Full-Text Search Extension to XQuery, In: Proceedings of WWW'04, 2004.

[3] Amer-Yahia, S., Lakshmanan, L. V. S., Pandit, S. (2004). FleXPath: Flexible Structure and Full-Text Querying for XML, In: Proceedings of SIGMOD' 04.

[4] Amer-Yahia, S., Curtmola, E., Deutsch, A. (2006). Flexible and Efficient XML Search with Complex Full-Text Predi 
cates, In: Proceedings of SIGMOD'06.

[5]Marian, A., Amer-Yahia,S., Koudas, N., Srivastava, D. (2005). Adaptive Processing of Top-K Queries in XML, In: Proceedings of ICDE'05, 2005.

[6] Amer-Yahia, S., Koudas, N., Marian, A., Srivastava, D., Toman, D. (2005). Structure and Content Scoring for $\mathrm{XML}$, In: Proceedings of VLDB'05.

[7] Sigurbjornsson, B., Kamps, J., Rijke, M. D. (2004). Processing Content- Oriented XPath Queries, In: Proceedings of CIKM'04, 2004.

[8] Xu, Y., Papakonstantinou, Y. (2005). Efficient Keyword Search for Smallest LCAs in XML Databases. SIGMOD 2005.

[9] Bloom, B. (1970). Space/Time Trade-offs in Hash Coding with Allowable Errors. Communications of the ACM. 13, July, p. 422-426.

[10] Grothoff , C. (2004). A Quick Introduction to Bloom Filters. Technical Report. Department of Computer Science, Purdue University, 2004.

[11] Bonifati, A., Matrangolo, U., Cuzzocrea, A., Jain, M. (2004). XPath Lookup Queries in P2P Networks, In: Proc. of WIDM 2004, p. 48-55, 2004.

[12] Cohen, S., Mamou, J., Kanza, Y., Sagiv, Y. (2003). XSEarch: A Semantic Search Engine for XML, In: Proc. of VLDB 2003, p. 45-56, 2003.

[13] Galanis, L., Wang, Y., Jeffery, S. R., and DeWitt, D. J. (2003). Locating Data Sources in Large Distributed Systems, In: Proc. of VLDB 2003, p. 874- 885, 2003.

[14] Graefe, G. (1993). Query Evaluation Techniques for Large Databases, ACM Computing Surveys. 25 (2) 73170, 1993.

[15] Guo, L., Shao, F., Botev, C., Shanmugasundaram, J. (2003). XRANK: Ranked Keyword Search over XML Documents, In: Proc. of SIGMOD 2003, p. 16-27, 2003.

[16] Chen, L., PapakonstantinouL, Y. (2012). Supporting Top-K Keyword Search in XML Databases, In: Proceedings of ICDE'10, 2010.
[17] Liu, S., Zou, Q., Chu, W. (2004). Configurable indexing and ranking for $X M L$ information retrieva, In: Proceedings of SIGIR'04, 2004.

[18] Han, Z., Le, J., Shen, B. (2006). Effectively Scoring for XML IR Queries, In: Proceedings of DEXA'06, 2006.

[19] Kaushik, R., Bohannon, P., Naughton, J. F., Shenoy, P. (2002). Updates for Structure Indexes, In: Proceedings of VLDB'02, 2002.

[20] Liu, F., Yu, C. T., Meng, W., Chowdhury, A. (2006). Effective Keyword Search in Relational Databases, In: Proceedings of SIGMOD'06.

[21] Buneman, P., Choi, B., Fan, W., Hutchison, R., Mann, R., Viglas, S. (2005). Vectorizing and Querying Large XML Repositories, In: Proceedings of ICDE'05, 2005.

[22] Sigurbjornsson, B., Kamps, J., Rijke, M. D. (2004). Processing Content-Oriented XPath Queries, In: Proceedings of CIKM'04, 2004.

[23] Xu, Y., Papakonstantinou, Y. (2005). Efficient Keyword Search for Smallest LCAs in XML Databases. SIGMOD 2005.

[24] Yates, R. B., Neto, B. R. (1999). Modern Information Retrieval, ACM Press, 1999.

[25] Zhang, C., Naughton, J., DeWitt, D., Luo, Q., Lohman, G. (2001).On Supporting Containment Queries in Relational Database Management Systems, In: Proceedings of SIGMOD'01, 2001.

[26] XMark. http://www.xml-benchmark.org/.

[27] XBench. http://se.uwaterloo.ca/'ddbms/projects/ xbench/

[28] XML Path Language (XPath) 2.0. http://www.w3.org/ TR/xpath20/

[29] XQuery 1.0: An XML Query Language. http:// www.w3.org/TR/xquery/

[30] DBLP. https://dblp.uni-trier.de/.

[31] SAX FOR .NET. http://saxdotnet.sourceforge.net/.

[32] XPathReader. https://www.microsoft.com/enus/ download/details. aspx?id=22677. 